\title{
QUALITY FACTORS AND ENERGY LOSSES OF SINGLE-CRYSTAL SILICON NANOWIRE ELECTROMECHANICAL RESONATORS
}

\author{
X.L. Feng", R.R. He ${ }^{1}$, P.D. Yang ${ }^{1}$, and M.L. Roukes \\ Kavli Nanoscience Institute, California Institute of Technology, Mail Code 114-36 \\ Pasadena, CA 91125, USA ( ${ }^{*}$ E-mail: xfeng@ caltech edu) \\ ${ }^{1}$ Department of Chemistry, University of California at Berkeley, Berkeley, CA 94720, USA
}

\begin{abstract}
We report measurements on quality factors ( $Q$ 's) of single-crystal silicon nanowire (SiNW) resonators operating in the very-high frequency (VHF) range. $Q$ 's of $\sim 200 \mathrm{MHz}$ metalized SiNWs are considerably lower than those of the pristine SiNWs operating at similar frequencies. The observed damping effect due to resonance transduction agrees well with the loaded- $Q$ model for magnetomotive scheme. The temperature dependency of dissipation $\left(Q^{-1}\right)$ is found to be approximately from $Q^{-1} \propto T^{0.3}$ to $Q^{-1} \propto T^{0.4}$. Clamping losses are becoming important for such VHF ultrasmall resonators.
\end{abstract}

Keywords: Nanoelectromechanical System, Nanowire, Resonator, Quality Factor $(Q)$, Dissipation

\section{INTRODUCTION}

Nanoelectromechanical systems (NEMS) with high resonant frequencies [1] offer potentials for high-resolution sensing [2] and high-speed signal processing [3] applications. Vibrating NEMS resonators are being actively developed via explorations in both top-down and bottom-up nanomechanical structures. We have recently demonstrated VHF SiNW resonators exhibiting attractive performance [4]. As the $Q$ 's are often critical for resonator applications, here we report measurements of $Q$ 's and analyses of energy losses in these SiNW resonators. .

\section{EXPERIMENTAL DATA AND ANALYSES}

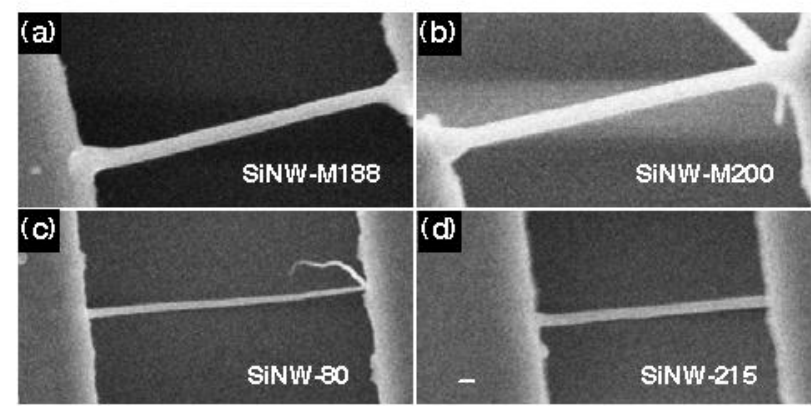

Figure 1. Scanning electron micrographs of VHF SiNW resonators. (a) Metalized $188 \mathrm{MHz}$ SiNW. (b) Metalized 200MHz SiNW. (c) Pristine $80 \mathrm{MHz}$ SiNW. (d) Pristine $215 \mathrm{MHz}$ SiNW. Scale bar in (d) is $100 \mathrm{~nm}$, for all the four micrographs.

Figure 1 shows images of the single-crystal SiNWs made by a bottom-up epitaxial process [5]. Measurements are performed with magnetomotive transduction, in vacuum within a cryostat, and at controlled temperatures, as detailed in [4].

An interesting observation is that the uncoated, pristine SiNWs exhibit much higher $Q$ 's than metalized ones do. We have measured $Q \approx 13100$ for SiNW-80, $Q \approx 5750$ for SiNW-215, $Q \approx 2500$ for SiNW-M188 and $Q \approx 2000$ for SiNW-M200, all at $T=25 \mathrm{~K}$ with $B=6$ Tesla. The SiNW-215 device has resonance frequency and dimensions in similar ranges as SiNW-M188 and SiNW-M200 do, but has a $Q$ of about $2 \sim 3$ times higher. This remarkable difference seems to be not solely from the geometry and dimension effects (such as those associated with aspect ratios and surface to volume ratios). This suggests that metallization may have introduced extra energy loss channels at the surface or material interfaces.

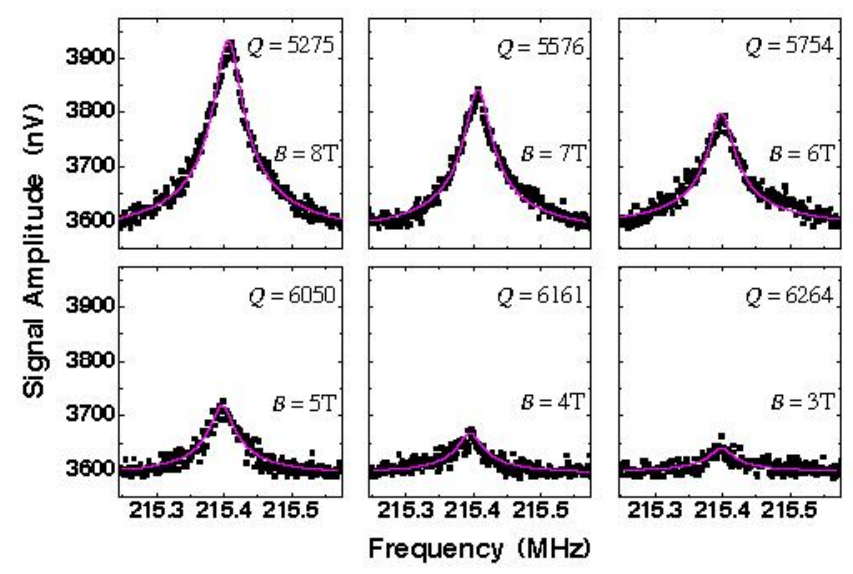

Figure 2. Q's of SiNW-215 at varied B field, extracted from optimized least-square nonlinear fitting of the measured resonance signals. 
As shown in Figure 2 with data from SiNW215 , the resonance amplitude decreases with lowered $B$ field, while the $Q$ increases. The $Q$ is obtained by optimizing the nonlinear fit of the resonance signal to a damped resonator model.

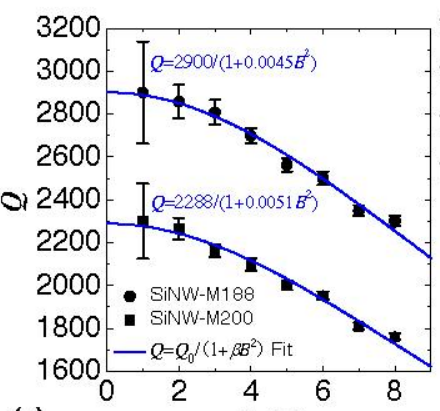

(a)

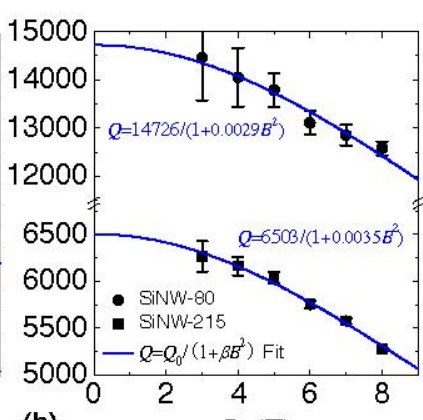

(b)

$B(\mathrm{~T})$
Figure 3. $Q$ as a function of the B field. (a) Data from two metalized SiNWs and (b) Very high Q's from two uncoated, pristine SiNWs. Solid lines are from fit to a loaded-Q model for the magnetomotive damping.

Figures 3 displays the measured $Q$ 's versus varied $B$ field for the four SiNWs. The decrease in measured $Q$ 's at higher $B$ field is due to magnetomotive (eddy-current) damping, which can be described by a loaded- $Q$ model [1] that indeed fits the data very well here.

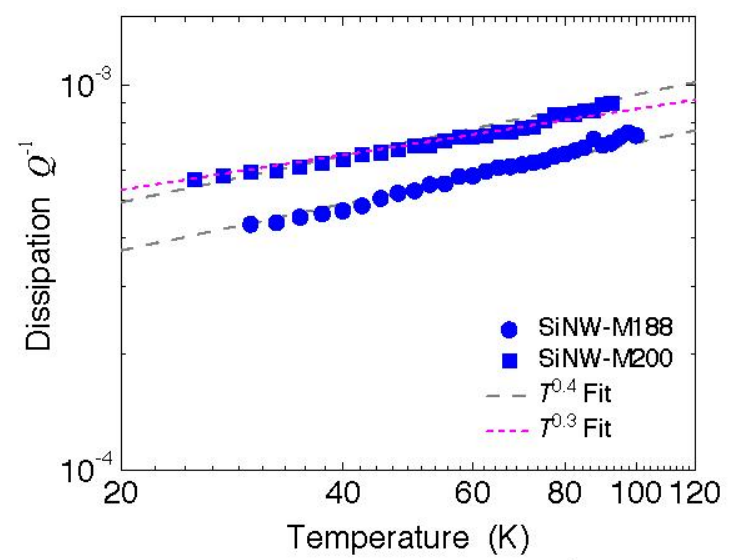

Figure 4. Measured dissipation $\left(Q^{-1}\right)$ in the two metalized SiNWs, as a function of temperature. A phenomenological dependence of about $Q^{-1} \propto T^{0.3-0.4}$ is observed in the range of $T=20 \sim 100 \mathrm{~K}$.

Figure 4 demonstrates the measured dissipation $\left(Q^{-1}\right)$ as a function of temperature, with data from two metalized SiNWs. The dissipation increases as the temperature is elevated, following a power law approximately from $Q^{-1} \propto T^{0.3}$ to $Q^{-1} \propto T^{0.4}$ in $T=20 \sim 100 \mathrm{~K}$ range. We believe the offset between the two data traces is because SiNW-M188 has less clamping losses than SiNW-M200 does, for the differences in their aspect ratios (18 and 16, respectively) and clamping structures (Figure 1). However, the $Q^{-1} \propto T^{\alpha} \quad(\alpha=0.3 \sim 0.4)$ power law seems to be not readily explained by geometric or surface effects. It may be related to some intrinsic dissipation processes in the single-crystal SiNW and/or metallization materials.

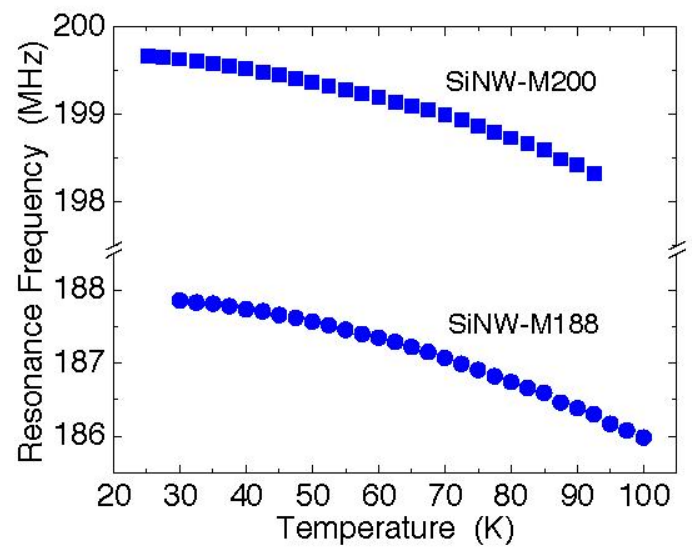

Figure 5. Resonance frequency as a function of temperature, data from SiNW-MI88 and SiNW-M200.

Figure 5 presents the calibrations of resonance frequency as the temperature is changed. The resonance frequency temperature coefficients of the metalized SiNWs are found to be about $50 \sim 60 \mathrm{ppm} / \mathrm{K}$ in the range of $T=20 \sim 100 \mathrm{~K}$.

\section{SUMMARY}

$Q$ 's of VHF SiNW resonators have been measured and analyzed. We aim to understand both the extrinsic energy losses such as the transduction damping effect, and the intrinsic dissipation mechanisms, to develop guidelines for $Q$-engineering for SiNW NEMS. In an extended work, we shall include data and modeling of the effects of geometry, surface, metallization, and intrinsic processes on the measured $Q$ 's.

\section{REFERENCES}

[1] X.M.H. Huang, X.L. Feng, M.L. Roukes, et al., New J. Phys., vol. 7, Art. No. 247, 2005.

[2] Y.T. Yang, C. Callegari, X.L. Feng, K.L. Ekinci, M.L. Roukes, Nano Lett., vol. 6, pp. 583-586, 2006.

[3] C.T.C. Nguyen, Proc. IEEE Freq. Contr. Symp., Vancouver, Canada, Aug. 29-31, pp. 1-11, 2005.

[4] X.L. Feng, R.R. He, P.D. Yang, M.L. Roukes, Nano Lett., vol. 7, 2007 (to be published).

[5] R.R. He, P.D. Yang, et al., Adv. Mater., vol. 17, pp. 2098-2 102, 2005. 